\title{
Serum Lipidomic Profiling in Breast Cancer to Identify Screening, Diagnostic, and Prognostic Biomarkers
}

\author{
Karima Fichtali, ${ }^{1}$ Amine Bititi, ${ }^{1}$ Adil Elghanmi, ${ }^{1}{ }^{*}$ and Bouchra Ghazi ${ }^{2}$
}

\begin{abstract}
Breast cancer is the major mortality cause of women worldwide. In the course of management of breast cancer, the identification of a biomarker is important in enhancing our knowledge on cancer pathology, predicting the response to treatment, and selecting the patients who are more favorable to receive certain treatments. These biomarkers have a prognostic value. In addition to traditional breast cancer prognosis factors such as the tumor size and grade, the axillary lymph node micrometastasis, and biomarkers such as HER2/neu, newly discovered biomarkers have been discovered. Some of these factors are genetic signature in tissue or in peripheral blood. Lipid profil, a simple and accessible biological examination, has been a novel path on the prediction of breast cancer risk of occurrence and recurrence in many studies. The main goal of our review is to evaluate lipid profile and breast cancer risk with an emphasis on the prognosis value of lipid profiles in breast cancer patient management.
\end{abstract}

Keywords: biomarkers; breast cancer; lipidomics; prognosis; serum lipid profile; statin

\section{Introduction}

Nowadays, breast cancer is the most commonly diagnosed life-threatening cancer in women and remains the leading cause of death by cancer among women, with 1.7 million cases and 521,900 deaths estimated in $2012 .^{1}$ Indeed, breast cancer accounts for $25 \%$ of all cancer cases and $15 \%$ of all cancer deaths in women. ${ }^{1}$ In a developing country, about $55.6 \%$ of all cancer cases and $62.1 \%$ of deaths are due to breast cancer. ${ }^{2}$ Interestingly, breast cancer incidence rates vary substantially by world region. ${ }^{3}$ Although there is a lower incidence of breast cancer in the North Africa region compared to Western countries, the global picture in Northern Africa is dynamically changing. ${ }^{4}$ Morocco, for example, is currently a country in epidemiological transition, and the breast cancer is becoming one of the main public health problems. ${ }^{5}$

The rising incidence tendency in low- and middleincome countries is mainly due to increases of risk factors caused by economic development and urbanization. Many factors among others include overweight and obesity, physical inactivity, earlier age at menarche, delayed childbearing, and/or having fewer children, as well as shorter duration of breastfeeding. ${ }^{6-8}$ Early screening, diagnosis, and management, together with improved treatments, may explain the international disparities in breast cancer mortality trends. Overall, there was an upward trend in the prevalence of overweight among Moroccan women, which increased over a period of 1 year from $29.0 \%$ in 1999 to $47.8 \%$ in $2000 .^{9}$

\footnotetext{
${ }^{1}$ Cheikh Khalifa International Hospital, Faculty of Medicine, Mohammed VI University of Health Sciences (UM6SS), Casablanca, Morocco.

${ }^{2}$ National Laboratory of Reference, Faculty of Medicine, Mohammed VI University of Health Sciences (UM6SS), Casablanca, Morocco.

*Address correspondence to: Adil Elghanmi, MD, PhD, Cheikh Khalifa International Hospital, Faculty of Medicine, Mohammed VI University of Health Sciences (UM6SS), Casablanca 82403, Morocco, E-mail: aelghanmi@um6ss.ma
}

(C) Karima Fichtali et al. 2020; Published by Mary Ann Liebert, Inc. This Open Access article is distributed under the terms of the Creative Commons License (http://creativecommons.org/licenses/by/4.0), which permits unrestricted use, distribution, and reproduction in any medium, provided the original work is properly cited. 
In Morocco, remarkable change in breast cancer incidence is largely due to improved diagnostic facilities and health care accessibility, as well as raising awareness of breast cancer.

The advanced stage at diagnosis in developing countries is contributed mainly to the lack of access to mammography, availability and quality of primary health care facilities, and the psychological and social impacts of treatments particularly for mastectomy. ${ }^{10,11}$ It is noteworthy that in 2003, a study conducted by the World Health Organization revealed that only $2.1 \%$ of Moroccan women aged 40 years or more had a clinical breast examination (or mammogram) in their life (Ministry of health, Morocco, 2007).

Nowadays, it is well established that the prognosis of most cancers is determined by the cancer stage at diagnosis, and women with early stage breast cancer have higher survival rates. ${ }^{10,12}$ Unfortunately, in Morocco like most developing countries, a very high proportion of breast cancer cases are diagnosed at late stages. ${ }^{13-16}$ The trends are completely different in western countries, in which the majority of patients with breast cancer are diagnosed at an early stage. For example, in the United States, it reached a high of $80 \%$ of cases. ${ }^{17}$

\section{Lipid Biomarkers in Breast Cancer}

The development and progression of cancer is a very complex multistep process involving reciprocal interactions of behavioral and environmental risk factors, dysregulated lipid homeostasis, and the cancer microenvironment. $^{18-20}$

Blood lipids, used for both adipose and glucose transportation, and lipoproteins, used for membrane production, have been incriminated in carcinogenesis through insulin resistance, inflammation, oxidative stress pathways, and the generation of signaling molecules in cancer cells. ${ }^{20-23}$ It has been shown that healthy lifestyle modifications for cancer prevention such as dietary changes, ${ }^{24,25}$ exercise, ${ }^{26}$ and smoking cessation $^{27}$ are effective to increase high-density lipoprotein (HDL) cholesterol (i.e., "good" cholesterol).

Dyslipidemia, a major component of metabolic syndrome, has been reported as playing a considerable role in the carcinogenesis of different cancers, like prostate cancer, ovarian cancer, and kidney cancer. ${ }^{28-30}$ Few well-documented metabolomics and lipidomics studies in breast cancer have shown the relation of lipids and breast cancer diagnosis and progression. ${ }^{31-33}$ Nevertheless, the focus of most of these studies has been total levels of lipids in cancer patients, and only a few of them were conducted with patients with benign breast diseases. In a recent comprehensive evaluation of plasma lipid profiles performed by Yang et al., including benign breast disease patients, it has also indicated the diagnostic efficiency of the lipid markers in breast cancer diseases. ${ }^{34}$

Presently it is well known that lipids are implicated in various ways in the pathogenesis of several human diseases, including breast cancer. ${ }^{35}$ In particular, the oncological potential of complex polar lipids may participate in development and metastasis of breast cancer. ${ }^{36}$ Recently, a panel of 15 plasma lipid species was identified as potential biomarkers for early diagnosis of breast cancer and was able to distinguish the early stage of breast cancer from benign lesions. ${ }^{37}$

Triglyceride levels, reflecting insulin and glycemia status, may also express the complex indirect effects of hyperglycemia and/or hyperinsulinemia on tumorpromoting pathways. ${ }^{38}$ The mechanism for the insulincancer link has been hypothesized to be mainly related to increased production of free insulin-like growth factor-1, a potent mitogen, and adipocyte-derived vascular endothelial growth factor, a key factor in tumor angiogenesis that leads to cell survival and migration. $^{38,39}$

Relatively sparse studies have evaluated the relationship between novel lipid biomarkers, such as apolipoprotein A-I (Apo A-I) and apolipoprotein B-100 (Apo B-100), and cancer risk. ${ }^{18}$ Apo A-I, the major protein components of HDL cholesterol, and Apo B-100, the major protein components of lowdensity lipoprotein (LDL) cholesterol, manifest the antiatherogenic and proatherogenic effect of HDL and LDL cholesterol, respectively. ${ }^{18}$ The prospective Swedish Apolipoprotein Mortality Risk study has shown no significant association of Apo B with colorectal cancer, but a significant positive association between triglycerides and colon cancer risk was found. ${ }^{40}$ There is no consistent information with regard to Apo A-I and breast cancer because studies are scarce with mixed results. ${ }^{41,42}$ In this issue, Han et al. showed that high Apo A-I levels were associated with higher risk of breast cancer. ${ }^{41}$ In the opposite, Chang et al. revealed a reduced risk of breast cancer with higher Apo A-I. ${ }^{42}$ In a large nested case-control study, measurement of plasma lipid marker concentrations at multiple times before the diagnosis of 
breast cancer showed decreased risk in breast cancer with higher Apo A-I. ${ }^{43}$

\section{Lipids as Early Diagnostic Biomarkers}

Early diagnosis plays an important role in the prognosis of patients with breast cancer. Mammography is the most reliable tool used for breast cancer scanning with the sensitivity at $54 \%$ to $77 \% .{ }^{44}$ However, the outcome of this screening tool is often not satisfactory because of the false-negative results and high false positive rate. ${ }^{45}$ In screening mammography, the over-diagnosis rate of breast cancer is variably quoted between $10 \%$ and $40 \%{ }^{46}$ Hence, abnormal screening mammograms engender additional expensive diagnostic tests, magnetic resonance imaging (MRI) and tissue sampling (fine needle aspiration, core biopsy, or excisional biopsy). Moreover, MRI has a high false-positive rate in differentiating breast lesions and may lead to a high frequency of useless biopsies of benign lesions, causing needless anxiety, physiological distress, and additional costs for patients. ${ }^{47}$ What worsens the situation is that about $10 \%$ of patients will be called back, from each screening examination for additional testing, but only $5 \%$ will be diagnosed with breast cancer. ${ }^{48}$ Thus, there is a pressing need for high accuracy technique and early diagnosis of malignant breast lesions, particularly for differentiating early cancer from benign lesions to avoid irrelevant, expensive, and invasive screening for benign patients.

Blood-based tumor markers are one approach to address these challenges. However, serum tumor markers are not yet used in clinical trials; for example, CA15.3 and BR27.29 are not used for breast cancer detection for their low sensitivity. ${ }^{49-52}$ In this issue, Min et al., performed the analysis on four different categories of phospholipids (phosphatidylserine [PS], phosphatidylinositol [PI], phosphatidylglycerol, and phosphatidic acid), from urine of breast cancer patients. ${ }^{31}$ Compared to healthy controls, in breast cancer patient group, two PS molecules (18:1/18:1 and 18:2/18:0) showed a significant increase; postoperative (after surgery) concentrations were reduced to normal levels. However, PI molecules (18:0/20:4) were significantly decreased in breast cancer samples. ${ }^{31}$ Chen et al., have identified a panel of plasma lipid species able to differentiate the early stage of breast cancer from benign lesions and act as relevant biomarkers for early diagnosis of breast cancer. ${ }^{37}$ This lipid profile was able to generate fast, high-efficiency, and high-throughput detection and involved only a minimally invasive procedure.
Lipidomics, a novel detection method, may serve as a promising prognostic evaluation of breast cancer especially in high-risk patient groups. Chen et al., have identified 15 lipid subtypes revealing significant differences of plasma concentration between breast cancer and benign patients. ${ }^{37}$ The plasma concentrations of phosphatidylcholine (PC) and ether-linked PC classes were observed to increase in patients with breast cancer. ${ }^{37}$ Exosomes derived from cells are rich in different components, such as heat shock proteins, annexin, lipids, and nucleic acids. ${ }^{53-55}$ Phuyal et al. have reported that an increase in cellular ether lipids (including PCs) affects the release and composition of exosomes in human prostate cancer PC-3 cells. ${ }^{56}$ In cancer patients, decreased levels of lysophosphatidylcholine (LPC) are linked with a high metabolic activity and activated inflammatory processes. ${ }^{57}$ LPCs have also been suggested to be a general indicator of disease severity. ${ }^{37,57}$ In breast cancer patients, the plasma concentration of LPC and cholesterol ester (CE) was noted to decrease, compared to that found in benign patients. ${ }^{37}$ The lower levels of LPCs seem to be associated with a higher metabolism rate in breast cancer patients. The repercussion of $\mathrm{CE}$ in breast cancer still has to be elucidated despite the association reported between $\mathrm{CE}$ and poor clinical outcome in human breast cancer. ${ }^{58}$

\section{Lipid Biomarkers in Prognosis of Breast Cancer}

Recent studies have reported the modification of lipid levels in prognosis of different types of cancers. ${ }^{59,60}$ For instance, reduction of cholesterol in oral cancers is alarming for the progression of cancerous lesions. ${ }^{61}$ Opposite results regarding the association between lipid levels and cancer progression seem to be due to the type of tumor and also by pathophysiological role of lipids related to cancer progression.

The association of lipids and lipoproteins with breast cancer was determined. ${ }^{62}$ Assessment of serum lipids among breast cancer, by Ghahremanfard et al., revealed highest levels of cholesterol and lipoproteins. ${ }^{63}$ Thereby, it has been concluded that increased serum lipid levels could have an important role in the progression of cancers in women, including breast and ovarian cancers, while lower level of lipids and lipoproteins may be used as markers for discriminating gastric cancer.

A higher consumption of fats increases circulating estrogen levels, increasing proliferation and cell damage, with direct effects on cancer growth. ${ }^{64}$ It has been found that there is an association between cholesterol and high HDL levels and increased risk of breast 
cancer. ${ }^{62,63}$ In another study, low HDL was correlated with increased postmenopausal breast cancer risk. ${ }^{65}$ Thereby, the association between serum HDL level and risk of breast cancer seems to be potentially influenced by menopausal status. By that, it has been reported that premenopausal patients have mean HDL levels lower than matched controls, while postmenopausal patients had higher levels than the controls. ${ }^{66,67}$ Interestingly, changes in serum lipid and lipoprotein profile are not only linked with the presence of some types of cancers but also there was a direct correlation between high LDL level and increased risk for lymph node metastasis. ${ }^{63}$

Potential prognostic value of lipidomic profile has been subjected to several investigations. It is commonly known that lipogenesis increased most solid tumors early on, with a potential impact on chemosensitivity of cancer cells. ${ }^{68}$ Hilvo et al. have investigated the association between the lipidomic profiles and pathologic complete response (pCR) in breast cancer patients receiving neoadjuvant chemotherapy. ${ }^{69}$ The results of this study have shown that serum triacylglycerol, containing C18:1 fatty acyl chains, was found at lower concentrations in the patients showing pCR. Formerly, Wei et al. achieved a serum metabolite analysis to identify a potential biomarker in response to neoadjuvant chemotherapy regimens against breast cancer (predictive power). ${ }^{70}$ Thus, metabolomic profiles of patients with pCR revealed significant differences in threonine, glutamine, and isoleucine levels; even more interestingly, in pCR patients, the free linoleic acid (C18:2) was detected in lower levels. ${ }^{70}$

\section{Statins and Breast Cancer Prognosis}

Nowadays, a lot of studies sustain the anticancer effects of HMG-CoA reductase inhibitors (statins). These studies do not support an association between statin use and reduced breast cancer incidence, but does support a protective effect of statins on breast cancer prognosis. Particularly, the simvastatin, a type of statins, may reduce the risk of breast cancer recurrence. ${ }^{71}$ Simvastatin inhibits DNA binding of the NF-kappa-B transcription factor, diminishes the expression of the antiapoptotic protein bcl-xL, and increases transcription of phosphatase and tensin homolog, which represses the oncogenic phosphatidylinositol-3-kinase pathway. ${ }^{72}$ Simvastatin accelerated the production of a variant of the $\mathrm{p} 53$ transcription factor and diminished the formation of bone metastases. ${ }^{73}$ This preventive effect would have a costeffective low-toxicity option for adjuvant therapy for breast cancer survivors. ${ }^{71}$

\section{Conclusion}

Breast cancer prognosis factors are an important component of the management process. They improve the selection of the appropriate treatment for a selected patient. Lipid profile is a novel prognosis factor as shown by multiple studies. It's a promising path in the prevention and better treatment of patients. Actually, medical literature suggests that diet and physical activity could influence survival. Moreover, statins are now used to enhance the positive prognosis of breast cancer. More studies are necessary for further evidence based guidelines.

\section{Author Disclosure Statement}

No competing financial interests exist.

\section{Funding Information}

No funding was received for this article.

\section{References}

1. Torre LA, Bray F, Siegel RL, et al. Global cancer statistics, 2012: global Cancer Statistics, 2012. CA Cancer J Clin. 2015;65:87-108.

2. Ferlay J, Steliarova-Foucher E, Lortet-Tieulent J, et al. Cancer incidence and mortality patterns in Europe: estimates for 40 countries in 2012. Eur J Cancer. 2013;49:1374-1403.

3. Ghoncheh M, Pournamdar Z, Salehiniya $H$. Incidence and mortality and epidemiology of breast cancer in the world. Asian Pac J Cancer Prev. 2016;17(S3):43-46.

4. Corbex M, Bouzbid S, Boffetta P. Features of breast cancer in developing countries, examples from North-Africa. Eur J Cancer. 2014;50:1808-1818.

5. Slaoui M, Razine R, Ibrahimi A, et al. Breast cancer in Morocco: a literature review. Asian Pac J Cancer Prev. 2014;15:1067-1074.

6. Boyle P, Howell A. The globalisation of breast cancer. Breast Cancer Res. 2010;12(Suppl 4):S7.

7. DeSantis CE, Bray F, Ferlay J, et al. International variation in female breast cancer incidence and mortality rates. Cancer Epidemiol Biomarkers Prev. 2015;24:1495-1506.

8. Porter P. "Westernizing" women's risks? Breast cancer in lower-income countries. N Engl J Med. 2008;358:213-216.

9. Toselli S, Gualdi-Russo E, Boulos DN, et al. Prevalence of overweight and obesity in adults from North Africa. Eur J Public Health. 2014;24(Suppl.1): 31-39.

10. Iqbal J, Ginsburg O, Rochon PA, et al. Differences in breast cancer stage at diagnosis and cancer-specific survival by race and ethnicity in the United States. JAMA. 2015;313:165-173.

11. Shulman LN, Willett W, Sievers $A$, et al. Breast cancer in developing countries: opportunities for improved survival. J Oncol. 2010;2010:595167.

12. Jazayeri SB, Saadat $S$, Ramezani $R$, et al. Incidence of primary breast cancer in Iran: ten-year national cancer registry data report. Cancer Epidemiol. 2015;39:519-527.

13. Amadou $A$, Torres-Mejía $G$, Hainaut $P$, et al. Breast cancer in Latin America: global burden, patterns, and risk factors. Salud Publica Mex. 2014;56:547-554

14. Kim Y, Yoo KY, Goodman MT. Differences in incidence, mortality and survival of breast cancer by regions and countries in Asia and contributing factors. Asian Pac J Cancer Prev. 2015;16:2857-2870.

15. Mathew A, George PS, Arjunan A, et al. Temporal trends and future prediction of breast cancer incidence across age groups in Trivandrum, South India. Asian Pac J Cancer Prev. 2016;17:2895-2899.

16. Schlichting JA, Soliman AS, Schairer C, et al. Breast cancer by age at diagnosis in the Gharbiah, Egypt, Population-Based Registry Compared to the United States Surveillance, Epidemiology, and End Results Program, 2004-2008. BioMed Res Int. 2015;2015:381574.

17. Mobley LR, Kuo TM. United States Health Policies and Late-stage Breast and colorectal cancer diagnosis: why such disparities by age? Health Econ Rev. 2015;5:58. 
18. Chandler PD, Song Y, Lin J, et al. Lipid biomarkers and long-term risk of cancer in the Womens Health Study. Am J Clin Nutr. 2016;103:13971407.

19. Baenke F, Peck B, Miess $H$, et al. Hooked on fat: the role of lipid synthesis in cancer metabolism and tumour development. Dis Model Mech. 2013;6: 1353-1363.

20. Ackerman D, Simon MC. Hypoxia, lipids, and cancer: surviving the harsh tumor microenvironment. Trends Cell Biol. 2014;24:472-478.

21. Giovannucci E. Metabolic syndrome, hyperinsulinemia, and colon cancer: a review. Am J Clin Nutr. 2007;86:s836-s842.

22. Currie E, Schulze A, Zechner R, et al. Cellular fatty acid metabolism and cancer. Cell Metab. 2013;18:153-161.

23. Gross RW, Han X. Lipidomics at the interface of structure and function in systems biology. Chem Biol. 2011;18:284-291.

24. Foster GD, Wyatt HR, Hill JO, et al. A randomized trial of a lowcarbohydrate diet for obesity. N Engl J Med. 2003;348:2082-2090.

25. Estruch R, Martínez-González MA, Corella D, et al. Effects of a mediterranean-style diet on cardiovascular risk factors: a randomized trial. Ann Intern Med. 2006;145:1-11.

26. Febbraio MA. Health benefits of exercise-more than meets the eye!: exercise metabolism in 2016. Nat Rev Endocrinol. 2017;13:72-74.

27. Criqui $\mathrm{MH}$, Wallace $\mathrm{RB}$, Heiss $\mathrm{G}$, et al. Cigarette smoking and plasma highdensity lipoprotein cholesterol. The Lipid Research Clinics Program Prevalence Study. Circulation. 1980;62(4 Pt 2):IV70-IV76.

28. Zhang GM, Qin XJ, Zhang HL, et al. Serum lipid profiles: novel biomarkers predicting advanced prostate cancer in patients receiving radical prostatectomy. Asian J Androl. 2015;17:239.

29. Liu Y, Chen Y, Momin A, et al. Elevation of sulfatides in ovarian cancer: an integrated transcriptomic and lipidomic analysis including tissueimaging mass spectrometry. Mol Cancer. 2010;9:186.

30. Cífková $E$, Holčapek $M$, Lísa $M$, et al. Lipidomic differentiation between human kidney tumors and surrounding normal tissues using HILIC-HPLC/ ESI-MS and multivariate data analysis. J Chromatr B Analyt Technol Biomed Life Sci. 2015;1000:14-21.

31. Min HK, Kong G, Moon MH. Quantitative analysis of urinary phospholipids found in patients with breast cancer by nanoflow liquid chromatography-tandem mass spectrometry: II. Negative ion mode analysis of four phospholipid classes. Anal Bioanal Chem. 2010;396:1273-1280.

32. Qiu Y, Zhou B, Su M, et al. Mass spectrometry-based quantitative metabolomics revealed a distinct lipid profile in breast cancer patients. Int J Mol Sci. 2013;14:8047-8061.

33. Hilvo M, Denkert $C$, Lehtinen $L$, et al. Novel theranostic opportunities offered by characterization of altered membrane lipid metabolism in breast cancer progression. Cancer Res. 2011;71:3236-3245.

34. Yang L, Cui X, Zhang N, et al. Comprehensive lipid profiling of plasma in patients with benign breast tumor and breast cancer reveals novel biomarkers. Anal Bioanal Chem. 2015;407:5065-5077.

35. Baumann J, Sevinsky C, Conklin DS. Lipid biology of breast cancer. Biochim Biophys Acta. 2013;1831:1509-1517.

36. Luo X, Cheng C, Tan Z, et al. Emerging roles of lipid metabolism in cancer metastasis. Mol Cancer. 2017;16:76.

37. Chen $X$, Chen $H$, Dai $M$, et al. Plasma lipidomics profiling identified lipid biomarkers in distinguishing early-stage breast cancer from benign lesions. Oncotarget. 2016;7:36622-36631.

38. Gialamas SP, Petridou ET, Tseleni-Balafouta S, et al. Serum adiponectin levels and tissue expression of adiponectin receptors are associated with risk, stage, and grade of colorectal cancer. Metabolism. 2011;60: 1530-1538.

39. Williams CJ, Mitsiades N, Sozopoulos E, et al. Adiponectin receptor expression is elevated in colorectal carcinomas but not in gastrointestinal stromal tumors. Endocr Relat Cancer. 2008;15:289-299.

40. Wulaningsih W, Garmo H, Holmberg $L$, et al. Serum lipids and the risk of gastrointestinal malignancies in the Swedish AMORIS study. J Cancer Epidemiol. 2012;2012:792034.

41. Han C, Zhang HT, Du L, et al. Serum levels of leptin, insulin, and lipids in relation to breast cancer in China. Endocrine. 2005;26:19-24.

42. Chang SJ, Hou MF, Tsai SM, et al. The association between lipid profiles and breast cancer among Taiwanese women. Clin Chem Lab Med. 2007; 45:1219-1223.

43. Martin LJ, Melnichouk O, Huszti E, et al. Serum lipids, lipoproteins, and risk of breast cancer: a nested case-control study using multiple time points. J Natl Cancer Inst. 2015;107:djv032.
44. Güth U, Huang DJ, Huber $M$, et al. Tumor size and detection in breast cancer: self-examination and clinical breast examination are at their limit. Cancer Detect Prev. 2008;32:224-228.

45. Gøtzsche PC, Jørgensen KJ. Screening for breast cancer with mammography. In: Cochrane Database of Systematic Reviews. The Cochrane Collaboration, ed. Hoboken, NJ: John Wiley \& Sons, Ltd.; 2013; DOI: 10.1002/14651858.CD001877.pub5

46. Morris E, Feig SA, Drexler M, et al. Implications of overdiagnosis: impact on screening mammography practices. Popul Health Manag. 2015; 18(Suppl. 1):S3-S11.

47. Yahalom J. Evidence-based breast cancer screening guidelines for women who received chest irradiation at a young age. J Clin Oncol. 2013; 31:2240-2242.

48. Rosenberg RD, Yankaskas BC, Abraham LA, et al. Performance benchmarks for screening mammography. Radiology. 2006;241:55-66.

49. Atahan K, Küpeli $H$, Gür $S$, et al. The value of serum biomarkers (Bc1, Bc2, $\mathrm{Bc3}$ ) in the diagnosis of early breast cancer. Int J Med Sci. 2011;8:148-155.

50. Donepudi MS, Kondapalli K, Amos SJ, et al. Breast cancer statistics and markers. J Cancer Res Ther. 2014;10:506-511.

51. Misek DE, Kim EH. Protein biomarkers for the early detection of breast cancer. Int J Proteomics. 2011;2011:343582.

52. Duffy MJ, Harbeck N, Nap M, et al. Clinical use of biomarkers in breast cancer: updated guidelines from the European Group on Tumor Markers (EGTM). Eur J Cancer. 2017;75:284-298.

53. Llorente A, Skotland T, Sylvänne T, et al. Molecular lipidomics of exosomes released by PC-3 prostate cancer cells. Biochim Biophys Acta. 2013;1831:1302-1309.

54. Kreimer S, Belov AM, Ghiran I. Mass-spectrometry-based molecular characterization of extracellular vesicles: lipidomics and proteomics. J Proteome Res. 2015;14:2367-2384.

55. Choi DS, Kim DK, Kim Y, et al. Proteomics, transcriptomics and lipidomics of exosomes and ectosomes. Proteomics. 2013;13:1554-1571.

56. Phuyal S, Skotland T, Hessvik NP, et al. The ether lipid precursor hexadecylglycerol stimulates the release and changes the composition of exosomes derived from PC-3 cells. J Biol Chem. 2015;290:4225-4237.

57. Taylor LA, Arends J, Hodina AK, et al. Plasma lyso-phosphatidylcholine concentration is decreased in cancer patients with weight loss and activated inflammatory status. Lipids Health Dis. 2007;6:17.

58. de Gonzalo-Calvo D, López-Vilaró L, Nasarre L, et al. Intratumor cholesteryl ester accumulation is associated with human breast cancer proliferation and aggressive potential: a molecular and clinicopathological study. BMC Cancer. 2015;15:460.

59. Cvetkovic Z, Cvetkovic B, Petrovic M, et al. Lipid profile as a prognostic factor in cancer patients. J BUON. 2009;14:501-506.

60. Muntoni S, Atzori L, Mereu R, et al. Serum lipoproteins and cancer. Nutr Metab Cardiovasc Dis. 2009;19:218-225.

61. Singh S, Ramesh V, Premalatha $B$, et al. Alterations in serum lipid profile patterns in oral cancer. J Nat Sci Biol Med. 2013;4:374.

62. Llanos AA, Makambi KH, Tucker CA, et al. Cholesterol, lipoproteins, and breast cancer risk in African American women. Ethn Dis. 2012;22:281-287.

63. Ghahremanfard F, Mirmohammadkhani M, Shahnazari B, et al. The valuable role of measuring serum lipid profile in cancer progression. Oman Med J. 2015;30:353-357.

64. Bertone ER, Rosner BA, Hunter DJ, et al. Dietary fat intake and ovarian cancer in a cohort of US women. Am J Epidemiol. 2002;156:22-31.

65. Furberg AS, Veierød MB, Wilsgaard T, et al. Serum high-density lipoprotein cholesterol, metabolic profile, and breast cancer risk. J Natl Cancer Inst. 2004;96:1152-1160.

66. Saha KR, Rahman MM, Paul AR, et al. Changes in lipid profile of postmenopausal women. Mymensingh Med J. 2013;22:706-711.

67. Moorman PG, Hulka BS, Hiatt RA, et al. Association between high-density lipoprotein cholesterol and breast cancer varies by menopausal status. Cancer Epidemiol Biomarkers Prev. 1998;7:483-488.

68. Perrotti F, Rosa C, Cicalini I, et al. Advances in lipidomics for cancer biomarkers discovery. Int J Mol Sci. 2016;17:E1992.

69. Hilvo M, Gade S, Hyötyläinen T, et al. Monounsaturated fatty acids in serum triacylglycerols are associated with response to neoadjuvant chemotherapy in breast cancer patients: oleic Acid and Response to Neoadjuvant Chemotherapy. Int J Cancer. 2014;134:1725-1733.

70. Wei S, Liu L, Zhang J, et al. Metabolomics approach for predicting response to neoadjuvant chemotherapy for breast cancer. Mol Oncol. 2013; 7:297-307. 
71. Ahern TP, Lash TL, Damkier P, et al. Statins and breast cancer prognosis: evidence and opportunities. Lancet Oncol. 2014;15:e461-e468.

72. Campbell MJ, Esserman LJ, Zhou Y, et al. Breast cancer growth prevention by statins. Cancer Res. 2006;66:8707-8714.

73. Mandal CC, Ghosh-Choudhury N, Yoneda T, et al. Simvastatin prevents skeletal metastasis of breast cancer by an antagonistic interplay between p53 and CD44. J Biol Chem. 2011;286:11314-11327.

Cite this article as: Fichtali K, Bititi A, Elghanmi A, Ghazi B (2020) Serum lipidomic profiling in breast cancer to identify screening, diagnostic, and prognostic biomarkers, BioResearch Open Access 9:1, 1-6, DOI: 10.1089/biores.2018.0022.

\section{Abbreviations Used}

Apo A-I = apolipoprotein A-I

Apo B-100 = apolipoprotein B-100

$\mathrm{CE}=$ cholesterol ester

$\mathrm{HDL}=$ high-density lipoprotein

$\mathrm{LDL}=$ low-density lipoprotein

LPC = lysophosphatidylcholine

$\mathrm{MRI}=$ magnetic resonance imaging

$\mathrm{PC}=$ phosphatidylcholine

$\mathrm{pCR}=$ pathologic complete response

$\mathrm{PI}=$ phosphatidylinositol

$\mathrm{PS}=$ phosphatidylserine

\section{Publish in BioResearch Open Access}

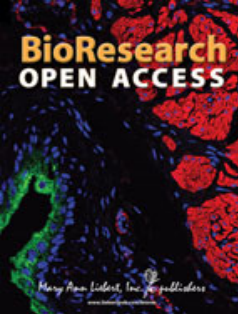

- Broad coverage of biomedical research - Immediate, unrestricted online access

- Rigorous peer review

- Compliance with open access mandates

- Authors retain copyright

- Highly indexed

- Targeted email marketing

liebertpub.com/biores 